University of Wollongong

Research Online

Faculty of Engineering and Information

Faculty of Engineering and Information

Sciences - Papers: Part A

Sciences

$1-1-2014$

Particle inertial focusing and its mechanism in a serpentine microchannel

Jun Zhang

University of Wollongong, jz218@uowmail.edu.au

Weihua Li

University of Wollongong, weihuali@uow.edu.au

Ming Li

University of Wollongong, ml433@uowmail.edu.au

Gursel Alici

University of Wollongong, gursel@uow.edu.au

Nam-Trung Nguyen

Griffith University

Follow this and additional works at: https://ro.uow.edu.au/eispapers

Part of the Engineering Commons, and the Science and Technology Studies Commons

Research Online is the open access institutional repository for the University of Wollongong. For further information contact the UOW Library: research-pubs@uow.edu.au 


\title{
Particle inertial focusing and its mechanism in a serpentine microchannel
}

\author{
Abstract \\ Particle inertial focusing in a curved channel promises a big potential for lab-on-a-chip applications. This \\ focusing concept is usually based on the balance of inertial lift force and the drag of secondary flow. This \\ paper proposes a new focusing concept independent of inertial lift force, relying solely on secondary flow \\ drag and particle centrifugal force. Firstly, a focusing mechanism in a serpentine channel is introduced, \\ and some design considerations are described in order to make the proposed focusing concept valid. \\ Then, numerical modelling based on the proposed focusing mechanism is conducted, and the numerical \\ results agree well with the experimental ones, which verify the rationality of proposed mechanism. Thirdly, \\ the effects of flow condition and particle size on the focusing performance are studied. The effect of \\ particle centrifugal force on particle focusing in a serpentine microchannel is carefully evaluated. Finally, \\ the speed of focussed particles at the outlet is measured by a micro-PIV, which further certifies the \\ focusing positions of particles within the cross section. Our study provides insights into the role of \\ centrifugal force on inertial focusing. This paper demonstrates for the first time that a single focusing \\ streak can be achieved in a symmetric serpentine channel. The simple serpentine microchannel can \\ easily be implemented in a single-layer microfluidic device. No sheath flow or external force field is \\ needed allowing a simple operation in a more complex lab-on-a-chip system.
}

\section{Keywords}

serpentine, microchannel, mechanism, its, focusing, inertial, particle

Disciplines

Engineering | Science and Technology Studies

\section{Publication Details}

Zhang, J., Li, W., Li, M., Alici, G. \& Nguyen, N. (2014). Particle inertial focusing and its mechanism in a serpentine microchannel. Microfluidics and Nanofluidics, 17 (2), 305-316. 


\title{
Particle inertial focusing and its mechanism in a serpentine microchannel
}

\author{
Jun Zhang, ${ }^{a}$ Weihua Li, ${ }^{* a}$ Ming Li, ${ }^{a}$ Gursel Alici ${ }^{a}$ and Nam-Trung Nguyen* ${ }^{b}$ \\ ${ }_{5}^{a}$ School of Mechanical, Materials and Mechatronic Engineering, University of Wollongong, Wollongong, NSW 2522, \\ Australia.E-mail: weihuali@uow.edu.au \\ ${ }^{b}$ Queensland Micro- and Nanotechnology Centre, Griffith University, Brisbane QLD 4111, Australia. E-mail: nam- \\ trung.nguyen@griffith.edu.au
}

\section{${ }_{10}$ Abstract}

Particle inertial focusing in a curved channel promises a big potential for lab-on-a-chip applications. This focusing concept is usually based on the balance of inertial lift force and the drag of secondary flow. This paper proposes a new focusing concept independent of inertial lift force, relying solely on secondary flow drag and particle centrifugal force. Firstly, a focusing mechanism in a serpentine channel is introduced, and some design considerations are described in order to make ${ }_{15}$ the proposed focusing concept valid. Then, numerical modelling based on the proposed focusing mechanism is conducted, and the numerical results agree well with the experimental ones, which verify the rationality of proposed mechanism. Thirdly, the effects of flow condition and particle size on the focusing performance are studied. The effect of particle centrifugal force on particle focusing in a serpentine microchannel is carefully evaluated. Finally, speed of focussed particles at the outlet is measured by Micro-PIV, which further certifies the focusing positions of particles within the cross 20 section. Our study provides insights on the role of centrifugal force on inertial focusing. This paper demonstrates for the first time that a single focusing streak can be achieved in a symmetrical serpentine channel. The simple serpentine microchannel can easily be implemented in a single-layer microfluidic device. No sheath flow or external force field is needed allowing a simple operation in a more complex lab-on-a-chip system.

\section{Introduction}

${ }_{25}$ Microfluidic technology has been a hot research topic since its emergence in the early 1980s. This technology provides significant advantages over conventional technologies, including (i) reduced sample and reagent volumes, (ii) fast sample processing, (iii) high sensitivity, (iv) low cost, (v) improved portability, and (vi) the potential to be highly integrated and automated to reduce human intervention (Bhagat et al. 2010a). A variety of techniques have been developed to process biological samples in the microfluidic format. According to the source of the manipulating force, they can be categorised as 30 active and passive techniques. Active techniques such as dielectrophoresis (DEP) (Çetin and Li 2011), magnetophoresis (MP) (Forbes and Forry 2012) and acoustophoresis (AP) (Wang and Zhe 2011) rely on an external force field, whereas passive techniques depend entirely on the channel geometry or intrinsic hydrodynamic forces, such as the mechanical filter (Ji et al. 2008), pinched flow fractionation (PFF) (Yamada et al. 2004), deterministic lateral displacement (DLD) (Huang et al. 2004) and inertial microfluidics (Di Carlo 2009). Generally, active techniques can provide a more precise control of ${ }_{35}$ target particles. However, they have drawbacks such as low throughput and the need for an external force field. In contrast, 
a microfluidic device based on a passive method is always very simple and has a considerably higher throughput. High throughput is especially necessary for applications of rare target particles, such as the diagnostics of circulating tumour cells (CTCs) (Cristofanilli et al. 2004). A large volume of sample needs to be processed to deliver consistent diagnostic results. As a passive technique, inertial microfluidics meets this requirement. Its working principle relies on particle inertial ${ }_{5}$ migration and the inertial effects of particle (centrifugal force) and fluid (secondary flow) (Di Carlo 2009). These forces are dominant at a high flow rate, suitable for a high throughput process.

Inertial migration is a phenomenon where randomly dispersed particles in the entrance of a straight channel migrate laterally to several cross-sectional equilibrium positions after a long enough distance (Segre 1961; Segre and Silberberg 1962). Two dominant forces are widely recognised as being responsible for this phenomenon: the shear gradient lift force ${ }_{10} F_{L S}$ acting down the velocity gradient towards the channel walls, and a wall induced lift force $F_{L W}$ directed towards the centreline of the channel. The balance of these two forces creates several equilibrium positions in the cross section. The net inertial lift force was derived by Asmolov based on the method of matched asymptotic expansions (ASMOLOV 1999), and then simplified as follows (Di Carlo 2009).

15

$$
\begin{gathered}
F_{L}=\frac{f_{L} \rho_{f} U_{m}^{2} a^{4}}{D_{h}^{2}} \\
\operatorname{Re}_{C}=\frac{\rho_{f} U_{m} D_{h}}{\mu}
\end{gathered}
$$

where $\rho_{f}, U_{m}$ and $\mu$ are the fluid density, maximum velocity and dynamic viscosity, respectively. The spherical particles have a diameter $a$. The hydraulic diameter $D_{h}$ of the channel is defined as $D_{h}=D$ for a circular channel ( $D$ is the diameter of the circular cross-section) or $D_{h}=2 w h /(w+h)$ for a rectangular channel ( $w$ and $h$ correspond to width and height of the rectangular cross-section). The lift coefficient $f_{L}$ of the net inertial lift force is a function of the position of the particles 20 within the cross-section of channel $x_{C}$, channel Reynolds number $\operatorname{Re}_{C}$ and particle size $a$ (Di Carlo 2009; Zhou and Papautsky 2013). In a straight channel, the lateral migration velocity $U_{L}$ of the particle and the minimum channel length $L_{\text {min }}$, which is required for particles to migrate to their equilibrium positions, can be derived by balancing the net inertial lift force and Stokes drag (Bhagat et al. 2009).

$$
\begin{gathered}
U_{L}=\frac{\rho_{f} U_{m}^{2} a^{3}}{6 \pi \mu D_{h}^{2}} \\
L_{\min }=\frac{3 \pi \mu D_{h}^{3}}{\rho_{f} U_{m} a^{3}}
\end{gathered}
$$

In order to modify and assist the inertial migration to reduce the length of the channel, curvature was introduced into the channel to provide a secondary flow (or Dean vortex). Compared with a straight channel, generally a curved channel has some advantages, including (i) improvement of collection purity due to an adjustment of equilibrium position of particles; (ii) a reduction of channel footprint for the lateral migration of particles due to the assistance of secondary flow to 30 accelerate lateral migration; and (iii) the equilibrium separation of particles based on different equilibrium positions of particles with various sizes (Di Carlo 2009). The reported curving geometry for inertial microfluidics includes spirals (Seo et al. 2007b, a; Kuntaegowdanahalli et al. 2009; Vermes et al. 2012; Bhagat et al. 2008; Wu et al. 2012), single arc (Yoon 
et al. 2008; Gossett and Carlo 2009; Oozeki et al. 2009), and a symmetric and asymmetric serpentine (Di Carlo et al. 2008; Gossett and Carlo 2009; Oakey et al. 2010; Di Carlo et al. 2007). Meanwhile, expansion-contraction array channel which can generate Dean-like vortex in the cross section was also proposed to focus and sort particles (Lee et al. 2009b; Park et al. 2009; Lee et al. 2011a; Moon et al. 2011; Zhang et al. 2013). Lee et al. (2009b) proposed an expansion-contraction array 5 microchannel to focus particles three-dimensionally with the assistance of a sheath flow. However, introduce of the sheath flow brings potential of dilution and contamination on bio-particle sample. And it also complicates the operation of the whole microfluidic system. So a sheath-less microfluidc system is more preferred. Bhagat et al. (2010b) presented a sheathless microfluidic focuser using a spiral microchannel. Based on this focuser, a low cost on-chip flow cytometer was developed. This on-chip flow cytometer was demonstrated to have a throughput of 2,100 particles/sec, which is far less

10 than the throughput of a conventional flow cytometer $\left(\sim 7 \times 10^{4}\right.$ particles/sec) (Eisenstein 2006). In order to increase the throughput of this on-chip flow cytometer to the order of conventional flow cytometer, a parallelization technology is usually needed, such as reported parallel channels (Hansson et al. 2012; Hur et al. 2010). However, it is not easy to design parallel spiral channels in microfluidics. For parallelization, microchannel with linear structure (such as straight or serpentine) is more suitable. Di Carlo et al. (2007) introduced an asymmetric serpentine channel to focus particles into one ${ }_{15}$ streak in 2-D (top view), and later this asymmetric serpentine channel was combined with a straight section to successfully focus particles in 3-D. The focusing performance was evaluated by standard flow cytometry method. The results showed that this device can operate with increasing effectiveness at higher flow rates and concentration of particles, which is ideal for high throughput analysis (Oakey et al. 2010).

Although significant achievements have been obtained using curved channels (Hou et al. 2013; Wu et al. 2012; Lee et al. 20 2011b), a complete and understandable particle focusing mechanism is still lacking (Gossett and Carlo 2009). In the reported previous works, focusing is normally regarded as the balance of secondary flow (Dean vortex) and inertial lift force in the cross section, but the importance of particle inertia (centrifugal force) is rarely considered (Kuntaegowdanahalli et al. 2009; Vermes et al. 2012; Russom et al. 2009; Gossett and Carlo 2009). The dimension of channel cross section is restricted $(a / D>0.07)$ in order to provide an effective inertial lift force, which increases the flow resistance and more ${ }_{25}$ power is needed to pump the particle suspension. So a microfluidic focuser independent of inertial lift force can release this restriction. The counter-rotating Dean vortex is prone to mix particles. It needs to be suppressed in the application of particle focusing. Moreover, there are no suitable criteria to evaluate the focusing efficiency and a proper design consideration for a curved channel. For example, a suitable expression such as Eq. 4 for a straight channel to determine the channel length for focusing particles in a curved channel is essential in the design process.

30 In this paper, we propose a new concept of inertial focusing in a serpentine channel, which is independent of the inertial lift force. The focusing process of particles in a serpentine channel is investigated in details through analytical analysis, numerical simulation and experiments. The focusing mechanism is first proposed, with some design considerations presented. Then, numerical modelling based on the proposed mechanism is conducted, and the numerical results are verified by the experiments. Thirdly, the effects of the Reynolds number and particle size on the focusing performance are ${ }_{35}$ studied. The weightiness of particle centrifugal force on particle focusing is investigated and carefully evaluated. Finally, the position and velocity of focussed particles at the outlet are measured by micro particle image velocimetry (PIV), which further verify the equilibrium positions of particles in the channel cross section.

\section{Focusing mechanism and design considerations}




\subsection{Focusing mechanism}

Figure 1a is a schematic view of particles focusing in a serpentine channel. Briefly speaking, particles are deflected into the channel centre after each period, due to the centrifugal force and secondary flow drag with alternating directions. And a final consequence is that particles are focused at the centre of channel after enough periods (top view). More detailed 5 mechanism is discussed in the following.


Fig. 1 Focusing mechanism of particles in a serpentine channel. (a) Schematic view of particles focusing in a serpentine channel. (b) The trajectory and speed of particles in a serpentine channel. The coloured curves are the dynamic trajectory of micro-particles, and the colour legend is the speed of particles. Particle trajectory is obtained by the numerical simulation. (c) The viscous drag $F_{D}$ in the cross section of the channel. (d) Schematic illustration 10 of centrifugal movement of single particle within one turn.

It is well known that when fluid is flowing in a channel with curvature (e.g. a serpentine channel), two counter-rotating 
vortices are generated due to the non-uniform inertia of fluid elements within the channel cross section (Di Carlo et al. 2008). Particles suspended in a fluid follow the streamlines due to viscous drag. The viscous drag $F_{D}$ can be calculated by Stokes law as:

$$
F_{D}=3 \pi \mu a\left(v_{f r}-v_{p r}\right)
$$

5 where $v_{f r}$ and $v_{p r}$ are radial velocity of fluid and particles respectively, as shown in Fig. 1c.

Additionally, the inertia of particles at the turns causes a centrifugal force $F_{\text {Cent }}$ (Lim et al. 2003; Mach et al. 2011).

$$
F_{\text {Cent }}=\left(\rho_{p}-\rho_{f}\right) \pi a^{3} v_{p t}^{2} / 6 r
$$

where $\rho_{p}$ and $v_{p t}$ are the density and tangential velocity of particles, respectively, and $r$ is the radius of particle orbit.

When the viscous drag $F_{D}$ acts as a centripetal force to balance the particle centrifugal force $F_{C e n t}$ within a turn, the ${ }_{10}$ trajectory of the particles will be a perfect circular curve. Unfortunately, the viscous drag $F_{D}$ in a real situation cannot always balance the centrifugal force $F_{C e n t}$ because in the entrance of the turn $\left(v_{p r} \approx 0\right), F_{D}$ directs from the inner corner to the outer corner, and the direction of $F_{C e n t}$ is determined by the sign of $\left(\rho_{p}-\rho_{f}\right)$. In our later experiments, particles are normally suspended in deionized (DI) water (unless otherwise specifically indicated), and $\rho_{p}>\rho_{f} \quad\left(\rho_{p}=1.05 \mathrm{~g} / \mathrm{ml}, \rho_{f}=1 \mathrm{~g} / \mathrm{ml}\right)$. Therefore, $F_{D}$ and $F_{C e n t}$ are pointing to the same direction, from the inner corner to the outer corner. Thus, the movement of ${ }_{15}$ particle within each turn is actually a centrifugal movement. The centrifugal movement of particles within one turn is illustrated schematically in Fig. 1d. Particles accelerate along the radial direction towards the outer corner until they finally exceed the fluid radial velocity. At this moment, the viscous drag $F_{D}$ changes to the opposite direction to compete with the centrifugal force. The particles continue to accelerate until two forces reach the final balance. Reaching this point of balance may take some time, and the particles could possibly have arrived at a new tangential position. This time scale ${ }_{20}$ should be very small because particle acceleration is proportional to $a^{-2}\left(10^{12} \mathrm{~m} / \mathrm{s}^{2}\right.$ scale for micro-sized particle). For simplicity, the acceleration process of particles is neglected. The particle radial velocity $v_{r}$ can be obtained by the balance of viscous drag $F_{D}$ and centrifugal force $F_{C e n t}$ :

$$
v_{p r}=v_{f r}+\left(\rho_{p}-\rho_{f}\right) a^{2} v_{p t}^{2} / 18 r \mu
$$

Two components contribute to the particle radial velocity: secondary flow $v_{f r}$ and particle centrifugal force $\left(\rho_{p^{-}}\right.$ $\left.{ }_{25} \rho_{f}\right) a^{2} v_{p t}{ }^{2} / 18 r \mu$. Here, we define a new parameter: particle relative radial velocity $v_{p r r}$, which is the relative velocity of particle and fluid elements along the radial direction. This is actually the migration velocity of the particles across the fluid streamline, and is the only contribution made by the particle centrifugal force for particle focusing:

$$
v_{p r r}=v_{p r}-v_{f r}=\left(\rho_{p}-\rho_{f}\right) a^{2} v_{p t}^{2} / 18 r \mu
$$

Since the sign of $v_{p r r}$ is positive as discussed above, particles migrate across the streamlines from the inner corner to the 30 outer corner with a relative migration velocity of $v_{p r r}$ at each turn. Furthermore, the speed of particles in the inner corner is much higher than the outer corner: $v_{p t 1}>v_{p t 2}$, and the radius of particle orbit in the inner corner is normally smaller than the outer corner: $r_{p 1}<r_{p 2}$, Fig. 1b. According to Eq. 8, the relative migration velocity $v_{p r r}$ is always faster at the inner corner than at the outer corner: $v_{\text {prr1 }}>v_{\text {prr2 }}$. Moreover, the inner and outer corners switch after each U-turn, with an alternate direction of relative migration velocity $v_{p r r}$. The overall effect is that particles are deflected towards the centre of the 
channel after each period, and the final result will be a focused streak in the centre of the channel at the outlet. That means the centrifugal force of the particles alone can successfully focus the particles into the centre of the channel, even without the assistance of secondary flow. The weightiness of the centrifugal force of the particles on particle focusing will be discussed in the following. It should be noted that the orbit of the particles in each corner is not a perfect circular curve, so ${ }_{5} r_{p 1}$ and $r_{p 2}$ are actually the average radius of the particles' orbit within each curve section.

The efficiency of focusing can be evaluated by the ratio of the distance that particles move perpendicular to the streamline to the distance along the streamline, and this ratio is equivalent to the ratio of particle velocity perpendicular and parallel to the streamline (Zhu et al. 2009). For focusing efficiency, the larger is this ratio $\delta$, the better.

$$
\delta=\frac{L_{r}}{L_{t}}=\frac{v_{p r}}{v_{p t}}=\frac{v_{f r}}{v_{p t}}+\frac{\left(\rho_{p}-\rho_{f}\right) a^{2} v_{p t}}{18 r \mu}
$$

10 The weightiness of the centrifugal force of the particles on particle focusing can be evaluated by the ratio of $v_{p r r}$ to $v_{p r}$. The larger is this ratio $\eta_{c e n t}$, the more significant is the centrifugal force on particle focusing.

$$
\eta_{c e n t}=\frac{v_{p r r}}{v_{p r}}=\frac{1}{18 v_{f r} r \mu /\left(\rho_{p}-\rho_{f}\right) a^{2} v_{p t}^{2}+1}
$$

Particles need to migrate transversely half of the channel width $w$ to focus into the centre of the channel, so the minimum arc length of the channel (focusing length) $L_{c \min }$ is:

15

$$
L_{c \min }=\frac{w v_{p t}}{2 \varepsilon\left(v_{f r}+\left(\rho_{p}-\rho_{f}\right) a^{2} v_{p t}^{2} / 18 r \mu\right)}
$$

where $\varepsilon$ is the correcting coefficient that takes into account the opposite effects of particle centrifugal force and secondary flow in the alternating turns where the inner and outer corners switch their positions. When particles are moving through the turn where the inner and outer corners switch, previous lateral (radial) displacement toward the centre of the channel (especially for particles in the previous inner corner) will be partially counteracted by the opposite centrifugal force and ${ }_{20}$ secondary flow in the new turn. Correction coefficient $\varepsilon$ is between 0 and 1 , and it should be a function of channel Reynolds number, particle size and channel dimension. When $\varepsilon=1$, the opposite centrifugal force and secondary flow in the alternating turns have no negative effects on particle focusing. When $\varepsilon=0$, focusing effects of centrifugal force and secondary flow have been completely counteracted by their alternating counterparts, and no focusing can be achieved.

In a curved channel, the magnitude of secondary flow is quantified by the Dean number $D e$.

$$
25 \quad D e=\operatorname{Re} \sqrt{\frac{D_{h}}{2 R}}
$$

where $R$ is the radius of channel curvature and Re is the flow Reynolds number which is defined based on average fluid velocity $U_{f}$ (Bhagat et al. 2008).

The velocity of secondary flow $U_{D}$ can be calculated as (Bhagat et al. 2010b; Vermes et al. 2012):

$$
U_{D}=1.8 \times 10^{-4} \times D e^{1.63}
$$

Substitute Eqs. 12 and 13 to Eqs. 9 11 and assuming $v_{f r}=U_{D}, v_{p t}=U_{f}$ and $r=R$, result in: 


$$
\begin{gathered}
\delta=1.8 \times 10^{-4} U_{f}^{0.63}\left(\frac{\rho_{f} D_{h}}{\mu} \sqrt{\frac{D_{h}}{2 R}}\right)^{1.63}+\frac{\left(\rho_{p}-\rho_{f}\right) a^{2} U_{f}}{18 R \mu} \\
\eta_{c e n t}=\frac{v_{p r r}}{v_{p r}}=\frac{1}{18.42 \times 10^{-4} \rho_{f}^{1.63} D_{h}^{2.445} R^{0.185} /\left(\rho_{p}-\rho_{f}\right) a^{2} U_{f}^{0.37} \mu^{0.63}+1} \\
\left.L_{c \min }=\frac{w\left(1.8 \times 10^{-4} \times U_{f}^{0.63} \times\left(\frac{\rho_{f} D_{h}}{\mu} \sqrt{\frac{D_{h}}{2 R}}\right)^{1.63}+\left(\rho_{p}-\rho_{f}\right) a^{2} U_{f} / 18 R \mu\right)}{2 \varepsilon(18)}\right)
\end{gathered}
$$

Based on above analytical analysis, one can easily determine the length of the serpentine channel (Eq. 16) to focus ${ }_{5}$ particles with certain size $a$ in the design process. It should be noted that the above analytical analysis only considers the effects of particle centrifugal force and secondary flow on the particle focusing process, and the effects of particle inertial migration phenomenon and mixing effects of secondary flow were neglected. In fact, inertial migration can play an important role for particle focusing, which was claimed as one of the dominant effects in a curved channel (Di Carlo et al. 2007). Inertial migration will become obvious when $a / D_{h}>0.07$, and even dominates particle behaviour when the particle ${ }_{10}$ Reynolds number $R_{p}\left(R_{P}=R e_{C} \times a^{2} / D_{h}{ }^{2}\right)$ is on the order of 1 (Di Carlo et al. 2007; Bhagat et al. 2008; Bhagat et al. 2010b). Also for the secondary flow, counter-rotating streamlines are prone to mixing particles by entraining them. Mixing effects must be inhibited to prevent particles from defocusing. In order to make our assumption valid, some design considerations for the channel structure need to be carefully addressed as follow.

\subsection{Suppression of mixing effects of secondary flow}

15 With the secondary flow, a counter-rotating vortex has the role of agitation and perturbation, which is beneficial for mixing (Stroock et al. 2002; Sudarsan and Ugaz 2006; Lee et al. 2009a) and heat transfer (Zheng et al. 2013), but not desirable for particle focusing because it tends to defocus and pull particles along its circulating streamlines. In order to suppress the mixing effect, a micro-channel with a low aspect ratio (AP) was suggested (Yoon et al. 2008), which was also verified by our numerical results (Fig. S1a b). For an extremely low aspect ratio channel (Fig. S1a(i)), the fluid velocity 20 along $z$ (vertical) direction is too small to drag particles vertically, so outward and inward streams are impossible to circulate particles in the cross-section. In our present work, the aspect ratio of the channel was set as $1 / 5$ (channel height=40 $\mu \mathrm{m}$, channel width=200 $\mu \mathrm{m}$ ), which is small enough to provide a wide available working area while inhibiting the mixing effects of secondary flow effectively (Fig. S1b(ii)). Additionally, the ratio of particle to channel size was critical for the exhibition of mixing effects. Yoon et al. (Yoon et al. 2008) demonstrated that particles smaller than 27\% of the ${ }_{25}$ channel height will obtain an inward velocity due to the mixing effects in a curved channel. However, due to the strong suppression on the circulating streamlines in an extremely low aspect ratio channel here, the ratio of particle diameter to channel height can actually be a little smaller than the theoretical value of $27 \%$. In our experiments, we found that this ratio can be as small as $20 \%$.

\subsection{Neglect of inertial lift force}

30 As we know, inertial migration becomes apparent when $a / D_{h}>0.07$ and $R_{p} \sim 1$. The number and position of the inertial equilibrium position depend mainly on the geometry of the channel. In a straight channel with $\mathrm{AP}=1$ as shown in Fig. S1c(i), there are four equilibrium positions, facing the centre of each channel surface (Di Carlo 2009). When AP is between 1/3 and 1/2 as shown in Fig. S1c(ii), the equilibrium positions are reduced to two, due to the blunted velocity profile along 
the long face of the channel and corresponding reduction of shear gradient lift force (Chung et al. 2013). However, by lowering the aspect ratio of the channel to $1 / 5$, as the inertial lift force is very weak along the long face, it was hard to observe very distinct equilibrium positions. The weak equilibrium positions of the particles varied under different flow conditions, as shown in Fig. S1c(iii v). In our experiments the maximum ratio of particle diameter to channel width was 5 0.065, which is still less than 0.07 . Therefore, inertial migration along the long face can be neglected, which is not expected to cause significant errors, and the particles along the short face will focus at the top and bottom of the channel due to the sharp parabolic velocity profile. Also in the following section, numerical simulation without the consideration of inertial lift force was conducted. The numerical results were then verified by experimental ones, which further indicated that the neglect of inertial lift force was reasonable.

\section{Materials and methods}

\subsection{Design and fabrication of the micro-channel}

Figure S2 (a) shows the structure of the serpentine channel used in our experiments. The channel consists of a $15.2 \mathrm{~mm}$ serpentine section with 15 periods. The depth of the channel is uniform at $40 \mu \mathrm{m}$. The length and width of each U-turn are both $700 \mu \mathrm{m}$. The device was fabricated by standard photolithography and soft lithography techniques. The detailed ${ }_{15}$ fabricating procedure was given elsewhere (Duffy et al. 1998).

\subsection{Particle suspension}

Internally dyed fluorescent polystyrene particles were purchased from Thermo Fisher Scientific. Particles with a diameter $a=8 \mu \mathrm{m}$ (Product No. 36-3, CV18\%), $9.9 \mu \mathrm{m}$ (Product No. G1000, CV5\%) and $13 \mu \mathrm{m}$ (Product No. 36-4, CV16\%) were suspended respectively in deionized (DI) water with $0.1 \% \mathrm{w} / \mathrm{v}$ Tween 20 (SIGMA-ALDRICH Product No. P9416), ${ }_{20}$ preventing the particles from aggregation. The weight ratio of particles in the suspension was $0.025 \% \sim 0.1 \%$.

\subsection{Experimental setup and method}

The microfluidic device was placed on an inverted microscope (CKX41, Olympus, Japan), illuminated by a mercury arc lamp. Particle suspension was pumped by a syringe pump (Legato 100, Kd Scientific), as shown in Figure S2 (b). The fluorescence images were observed and captured by a CCD camera (Rolera Bolt, Q-imaging, Australia), and then post${ }_{25}$ processed and analysed using the software Q-Capture Pro 7 (Q-imaging, Australia). The exposure time for each frame was set at $100 \mathrm{~ms}$. The streak width was determined by measuring the distance between points where the intensity profile crossed the $50 \%$ threshold. The streak position was taken as the middle of the $50 \%$ threshold intensity. Focusing was achieved when the streak width became less than 2 times the diameter of the particles (Martel and Toner 2012). A microPIV (TSI, USA) system was also used to capture snapshots of fluorescent particles at the outlet, and to evaluate the 30 focusing performance more specifically. The space and speed of the particles at the outlet were obtained by analysing the snapshot pairs.

\subsection{Numerical simulation}

In order to understand and predict the focusing behaviour of the particles, numerical modelling was used to calculate the flow field and trajectory of the particles in the serpentine channel. A laminar steady incompressible flow model was used to ${ }_{35}$ calculate the flow field. A non-slip boundary condition was applied onto the surfaces of the channel, and then the calculated flow field was used to trace the particles with the mass model in COMSOL (Burlington, MA) to predict particle 
trajectory in the serpentine channel. Equations governing steady incompressible flow are:

N-S equation:

$$
\rho_{f} \vec{v}_{f} \cdot \nabla \vec{v}_{f}=-\nabla P+\mu \nabla^{2} \vec{v}_{f}
$$

Continuity equation:

$$
\nabla \cdot \vec{v}_{f}=0
$$

Non-slip boundary condition:

$$
\vec{v}_{w}=0
$$

where $\vec{v}_{f}$ and $P$ are the velocity vector and pressure of fluid, respectively; $\vec{v}_{w}$ is fluid velocity vector at the channel walls. $\nabla$ is Nabla operator: $\nabla=\vec{i} \frac{\partial}{\partial x}+\vec{j} \frac{\partial}{\partial y}+\vec{k} \frac{\partial}{\partial z}$, and $\nabla^{2}$ is Laplace operator: $\nabla^{2}=\nabla \cdot \nabla=\frac{\partial^{2}}{\partial x^{2}}+\frac{\partial^{2}}{\partial y^{2}}+\frac{\partial^{2}}{\partial z^{2}}$.

The governing equation for particles' movement is:

$$
\begin{gathered}
m_{p} \ddot{\vec{X}}=\vec{F}_{\text {drag }} \\
F_{\text {drag }}=\frac{\pi}{4} a^{2} \rho_{f} v_{t}^{2}\left(1.84 \operatorname{Re}^{\prime-0.31}+0.293 \mathrm{Re}^{0.06}\right)^{3.45} \\
\operatorname{Re}^{\prime}=v_{t} \rho_{f} a / \mu \\
v_{t}=v_{f}-v_{p}
\end{gathered}
$$

Instead of stokes drag equation, here Khan and Richardson equation is used for calculation of viscous drag $F_{d r a g}$, as it has a wider agreement with experimental data compared to stokes drag (Richardson et al. 2002).

\section{Results and discussion}

In order to verify the feasibility of neglecting the inertial lift force for particle focusing in a serpentine channel, we ${ }_{20}$ conducted a numerical modelling which only took account of particle inertia and fluid viscous drag on particle focusing. The predicted trajectory of particles agreed well with particle streak in the experiments, which validated the proposed focusing concept. Then, the effects of flow condition and particle size on focusing performance were investigated. The effect of particle centrifugal force on particle focusing was investigated by suspending particles in solution with different densities. Finally, the speed of focussed particles at the outlet was measured by a micro-PIV system, and the results further 25 verified the particles positions in the cross section.

4.1 Validation of focusing concept and determination of correction coefficient $\varepsilon$

Figures 2a and 2b illustrated trajectory of particles in a serpentine microchannel obtained from numerical simulation, and its corresponding streak images from experiments were plotted in Fig. 2c. Focusing process between 1st and 5th periods can be found in Fig. S3. Note that the simulation results agreed very well with the experimental ones, as the randomly 
dispersed particles at the inlet shifted into the centre of the channel after each turn and the width of their streak decreased continuously, and finally they focused at the centre of channel at the outlet. It indicates that the proposed focusing concept which only takes account of particle inertia and secondary flow drag is reasonable. Although inertial lift force is still present in the actual situation, its effect is negligible compared to other two effects.

(a)

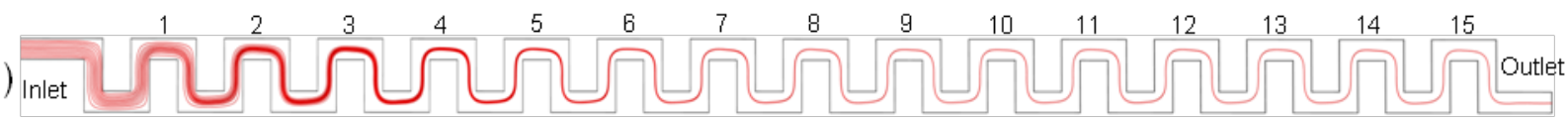

(b)
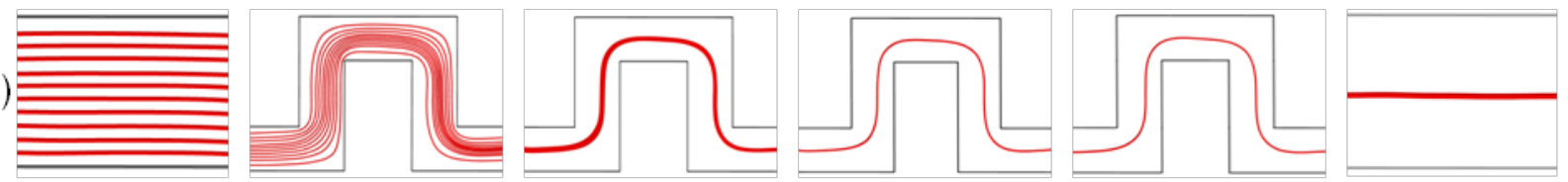

(c)

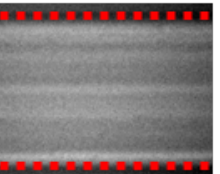

Inlet

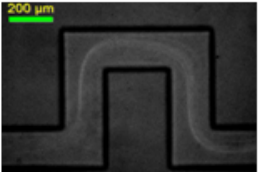

1 st

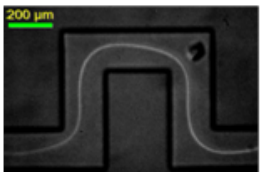

5th

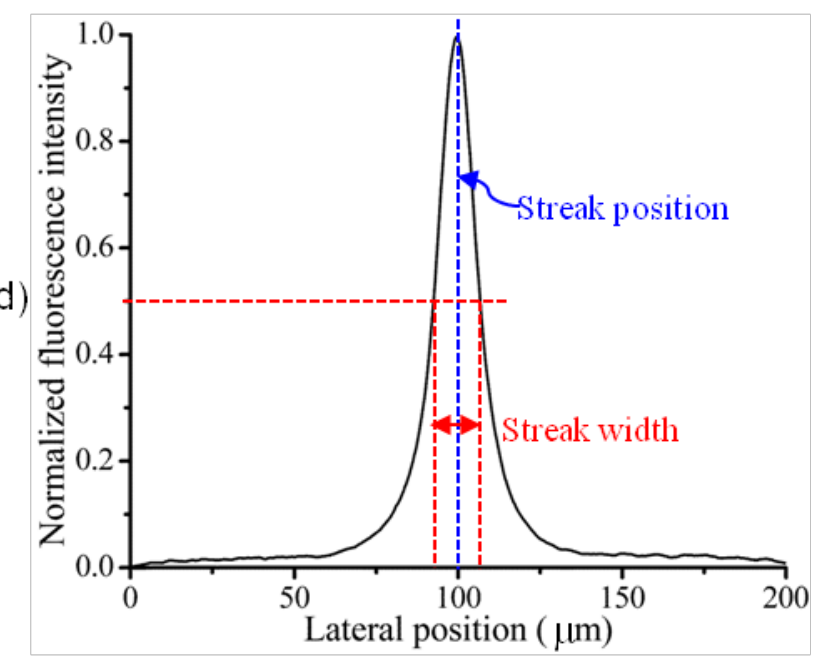

(i)

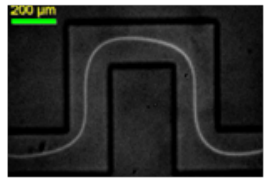

10th

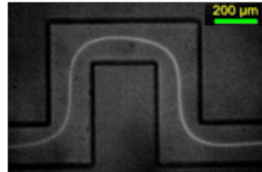

15th

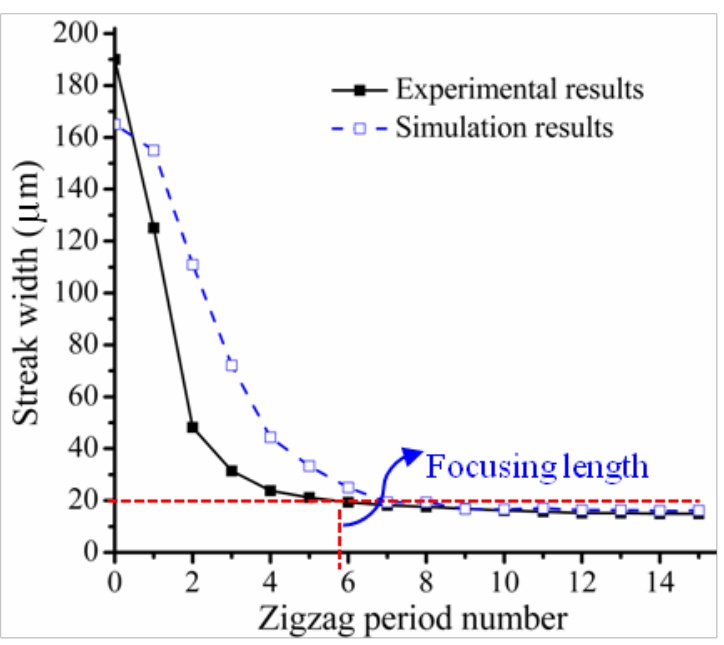

(ii)

Fig. 2 (a) The overview of particles focusing in a serpentine channel. (b) The numerical results of particles' trajectory at the inlet, typical zigzag periods and outlet of a serpentine channel. (c) The experimental streak images of fluorescent particles in the corresponding positions of the serpentine channel. (d) Particle streak width and position. (i) Determination of particle streak width and streak position from fluorescence intensity profile. (ii) Particle streak 10 width under different numbers of zigzag turns. The input fluid average velocity is $1.1 \mathrm{~m} / \mathrm{s}$, corresponding to the channel Reynolds number of 110 . Particle diameter is $9.9 \mu \mathrm{m}$.

The width of the particle streak under different numbers of zigzag periods was plotted in Fig. 2d. The width was determined by measuring the distance between two points where the intensity profile crossed the $50 \%$ threshold. The streak ${ }_{15}$ position was taken as the middle of the $50 \%$ threshold intensity (Fig. 2d(i)). The width of the particle streak decreased rapidly and particle focusing was achieved after the fifth zigzag period (Fig. 2d(ii)), with an arc length (focusing length) of about $11 \mathrm{~mm}$ when the channel Reynolds number was 110. In our experiments, best focusing happened when the channel Reynolds number was 160 and focusing length was only $3.75 \mathrm{~mm}$. This focusing length is shorter than most of the focusing lengths reported in the literature, and it even reached the level of state-of-the-art asymmetric curving channels (Di Carlo et 
al. 2007). The mean and standard deviation of the lateral positions of particle streak were determined by fitting the counts to a Gaussian distribution, and the particle streak lateral position was $99.0 \pm 0.97 \mu \mathrm{m}$, perfectly within the centre of the channel. Furthermore, compared with the asymmetric curving channel reported previously, our serpentine channel is much simpler because the radii of a larger curvature and a smaller curvature are different in an asymmetric curving channel and

5 must be determined respectively, whereas only one parameter needs to be selected in a symmetric serpentine channel. Furthermore, particles are focused at the centre of the channel which eliminates the difficulty of aligning the detection unit if our serpentine channel acts as a focusing unit for on-chip flow cytometry.

The correction coefficient $\varepsilon$ in Eq. 11 or 16 was calculated under several typical Reynolds numbers in the experiments, where the Reynolds numbers 110, 120, 140, and 160 correspond to a focusing length of about $10.8 \mathrm{~mm}, 6.75 \mathrm{~mm}, 4.75$ ${ }_{10} \mathrm{~mm}$, and $3.75 \mathrm{~mm}$, respectively. According to Eq. 16, $\varepsilon=0.258,0.388,0.504$ and 0.578 respectively (More detailed information is shown in Eq. S4). The correction coefficient $\varepsilon$ increases with an increasing channel Reynolds number because of the fast lateral migration velocity of particles at high Reynolds numbers, and there is less chance for opposite centrifugal force and secondary flow drag to deflect particles into two side walls in the alternating turns.

\subsection{Effects of the channel Reynolds number}

${ }_{15}$ From Eq. 14, the focusing efficiency is proportional to the input fluid velocity (or channel Reynolds number). In the experimental validation, the width of the particle streak was measured at the outlet under each flow condition, and the results were shown in Fig. 3a. The width of the streak decreases sharply with increasing Reynolds number, and after a critical value (defined as critical channel Reynolds number for particle focusing $\mathrm{Re}_{C C}$ ), focusing was achieved. The available working area on the $\mathrm{Re}_{c}-a$ space for particle focusing can be obtained. It is noted that this critical value was ${ }_{20}$ different for various particle sizes. This difference can be used to characterise the effects of particle size on focusing performance, which will be discussed in the following section in more detail.

To evaluate particle focusing more specifically, a micro-PIV system was used to capture snapshot images of fluorescent particles at the end of the last zigzag period, and the results were compared with the images of particle streaks observed from the fluorescence microscope (Fig. 3b). Particles are distributed within the streak and migrate into the centre when the ${ }_{25}$ Reynolds number has increased. This agrees well with the results from the streak images. However, when the channel Reynolds number exceeds the critical value for particle focusing, it is hard to evaluate the particle focusing more specifically by fluorescence streak image, such as particle position, particle speed, or the defection of certain particles within the particle chain. Perhaps a high speed camera (such as Photron SA-3, United Kingdom (Vermes et al. 2012), and Phantom v7.3, Vision Research, Inc (Gossett and Carlo 2009)) could be effective to analyse particle focusing in a more so detailed manner. 


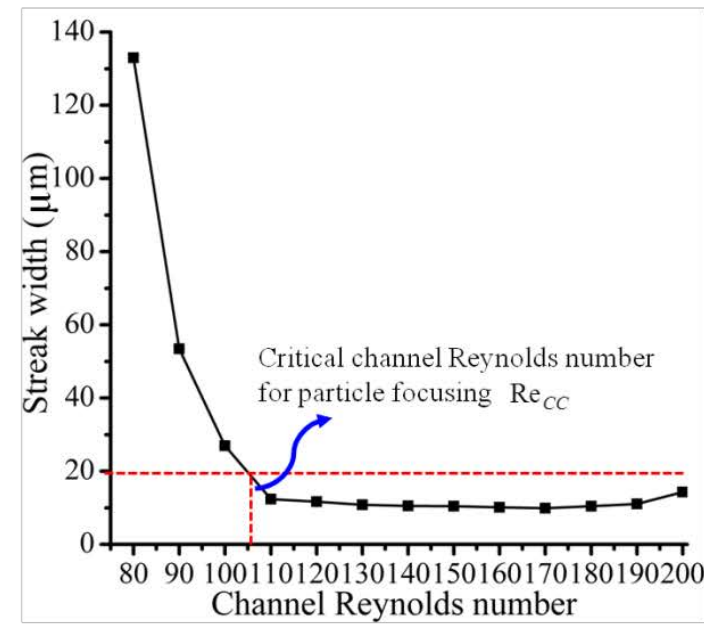

(a)
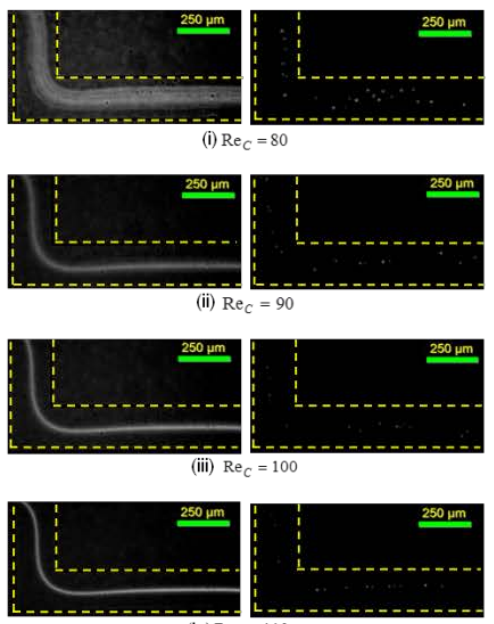

(iv) $\operatorname{Re}_{\mathrm{C}}=110$

(b)
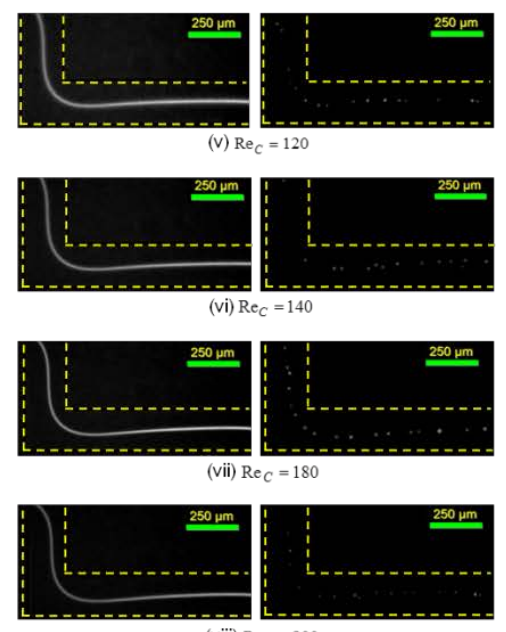

(viii) $\operatorname{Re}_{C}=200$

Fig. 3 (a) The particle streak width under different flow conditions (channel Reynolds number). (b) Particle streak images observed from fluorescence microscope (left) and particles snapshot image obtained from micro-PIV system (right) under different flow conditions.

\section{${ }_{5} 4.3$ Effects of particle size and particle inertia}

Equations 11 and 16 indicate that particles with a larger diameter can achieve focusing within a shorter arc length (focusing length) when the input flow condition (Reynolds number $R e_{C}$ ) is constant, but determining the critical arc length for focusing under each flow condition is not easy. Also, it is more important to know the available working area for particle focusing in a specific microfluidic device rather than the position where focusing occurs. Therefore, instead of a critical arc 10 length for focusing, the critical Reynolds number $\operatorname{Re}_{C C}$ (Fig. 3a) was used to evaluate the effects of particle size on focusing efficiency. Larger particles are expected to achieve focusing at a lower Reynolds number within the same arc length. This hypothesis was verified by the experimental results in Fig. 4. Fig. 4 also shows that larger particles can achieve focusing in a much wider available working area than smaller particles. The data is limited within the channel Reynolds number of 200, which corresponds to a flow rate of $1 \mathrm{ml} / \mathrm{min}$ (fluid average velocity $2 \mathrm{~m} / \mathrm{s}$ ). The reasons why the experiments stop at ${ }_{15}$ this flow rate are (i) sealing failure and liquid leakage could happen when the flow rate is too large, and because the pressure within the microfluidic chip is too high, and (ii) the counter-rotating secondary flow is becoming strong enough to begin to mix and defocus particles (Fig. S5).

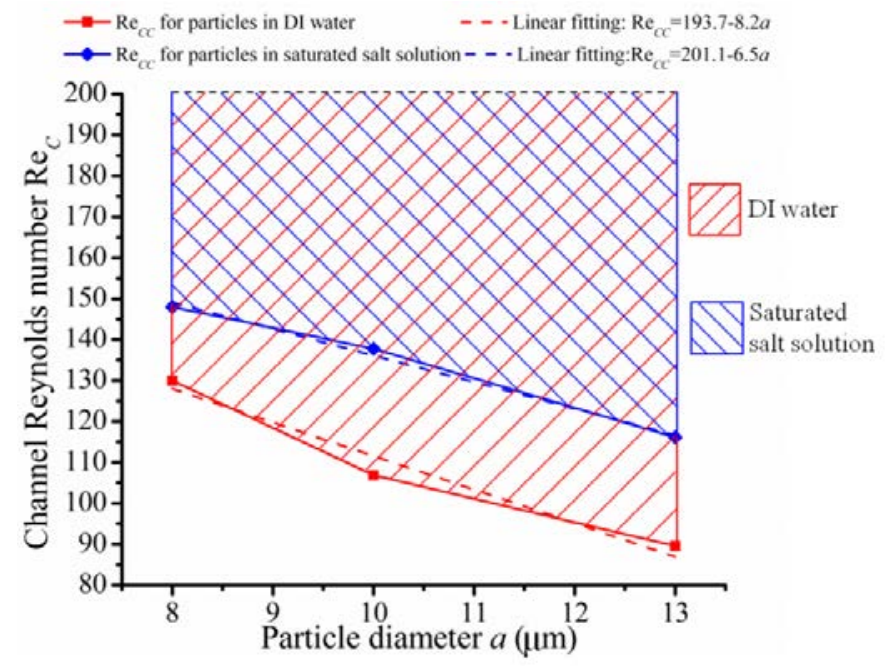


Fig. 4 The effects of particle size and solution density on the critical channel Reynolds number for particle focusing.

In addition, $8 \mu \mathrm{m}, 10 \mu \mathrm{m}$, and $13 \mu \mathrm{m}$ particles were also respectively prepared in a saturated salt solution $(\rho=1.20 \mathrm{~g} / \mathrm{ml})$, and then used to study the effects of solution density on particle focusing. As known from equation (6), the centrifugal ${ }_{5}$ force of the particles will change to the opposite direction when particles are not as dense as the solution. If the centrifugal force of the particles is stronger than the secondary flow drag, particle focusing will disappear, but particle focusing still can be observed in our experiments. This observation indicates that secondary flow is more important than particle centrifugal force in the particle focusing process. However, unlike the previous description that particle focusing is independent of solution density (particle centrifugal force) (Di Carlo et al. 2007; Russom et al. 2009), particle centrifugal ${ }_{10}$ force actually has a significant impact on particle focusing, although it is not a dominant effect. The critical channel Reynolds number for these particles in a saturated salt water solution is much higher than its corresponding value in a solution of DI water, meaning that the opposing centrifugal force can actually hinder particle focusing and decreases its available working area.

More specifically, the contribution of particle centrifugal force on particle focusing can be evaluated by $\eta_{\text {cent }}$ (Eq. 15), as ${ }_{15}$ discussed above. For $10 \mu$ m polystyrene particles in DI water, when $\operatorname{Re}_{C C}=110 \sim 200, \eta_{\text {cent }}=3.4 \% \sim 4.2 \%$, but for particles with a higher density (such as silica or metal), the weight of particle centrifugal force will be much higher. Taking a silica $\left(\mathrm{SiO}_{2}\right)$ particle for example, $\rho_{\text {silica }}=2.65 \mathrm{~g} / \mathrm{ml}$, with other parameters the same as the polystyrene particle suspension, the weight of particle centrifugal force on particle focusing can be as high as $\eta_{\text {cent }}=53.7 \% \sim 59.1 \%$. So the effect of solution density and particle centrifugal force needs to be considered carefully in any practical application. In addition, the fluid 20 viscosity is expected to influence the focusing behaviour too. According to Eq. 14 and 16, fluid with high viscosity will hinder the focusing theoretically. However, the flow field varies when fluid viscosity changes, which will certainly cause disturbance on inertial focusing process, at present we are not sure whether it is a positive or negative effect. So a better way is through experiments, there are two difficulties when conducting such an experiment: (i) choose or prepare a set of fluid solutions, which have different viscosities, but with same (or very close) density; (ii) For fluid with very high ${ }_{25}$ viscosity, it needs more power to pump the particle suspension into the microfluidic device, so a proper pumping system needs to be considered. After all, it will be the future work.

4.4 Position and velocity of particles at the outlet

A micro-PIV system was used to evaluate particle focusing more specifically because particle position and particle velocity are additional important information which can be used to characterise the quality of particle focusing. The results were so plotted in Fig. 5a. Particles were focused at the centre of the channel at the outlet, and the longitudinal position of the particles was not uniform, as expected in the real situations, with some particles deflecting from the particle chain. This was mainly due to the large deviation of particle size (CV16\%). Also the particles were not uniform in the suspension in practice because their density was slightly more than the solution density (DI water), and they were gradually settling down in the container and the micro-channel. It was also found that particle space is generally independent of the flow conditions. 


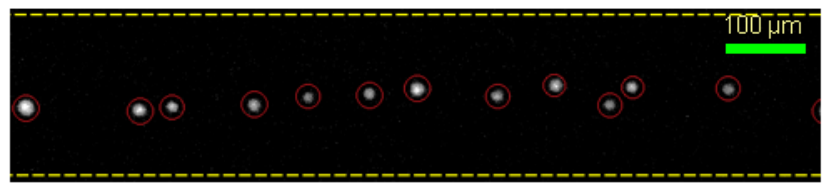

$\operatorname{Re}_{C}=120$

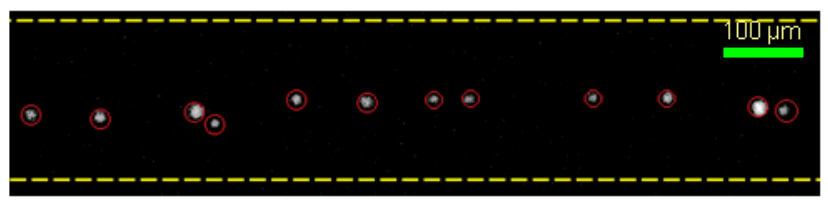

$\operatorname{Re}_{C}=140$



$\operatorname{Re}_{C}=160$

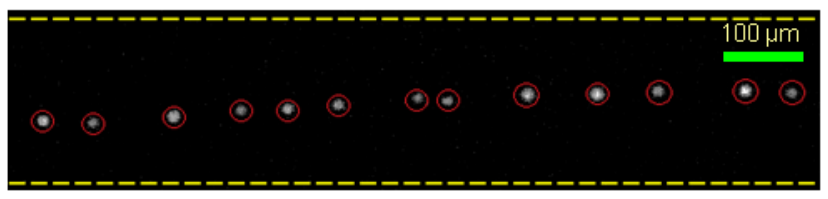

$\operatorname{Re}_{C}=180$

(a)

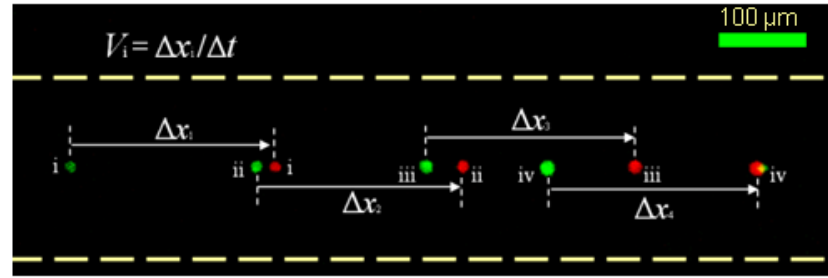

(i)

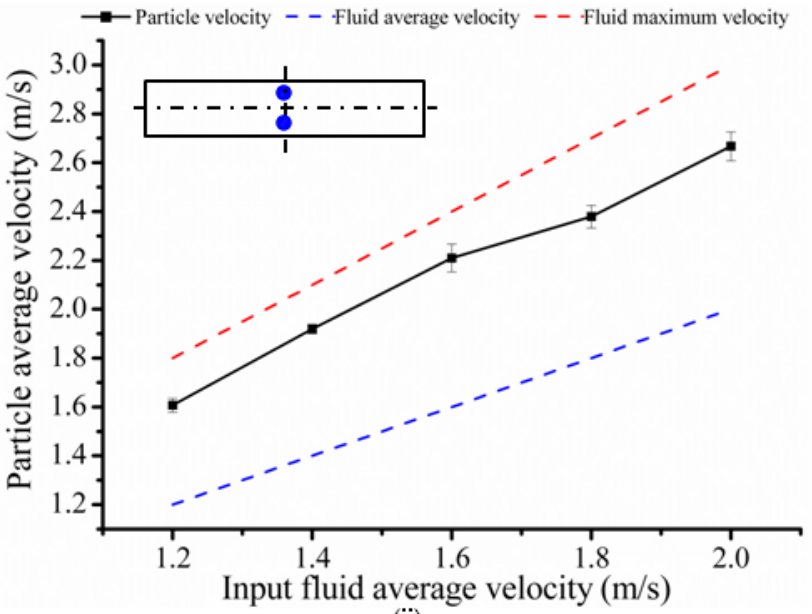

(ii)

(b)

Fig. 5 (a) Particle longitudinal space under different flow conditions. Particles are highlighted with red circle. (b) The particle velocity at the outlet. (i) Particle velocity is calculated as particle displacement divided by residence time $\Delta t(100 \mu \mathrm{s})$. Particle positions at two moments are superposed with two different colours. Green symbol is particles at the initial moment, and red symbol is particle position after $\Delta t$. (ii) Particle average velocity with respect to

5 input fluid velocity. Particle average velocity is calculated as $\left(\sum_{i=1}^{n} \Delta x_{i} / \Delta t\right) / n$. Two blue dots are schematic position of particles within channel crosssection at the outlet. Fluid maximum velocity is calculated as $U_{m}=1.5 U_{f}$ (Di Carlo et al. 2007).

Besides, the velocity of the particles was calculated as their displacement divided by their residence time (Fig. 5b(i)), and its average value was plotted against the input fluid velocity (Fig. 5b(ii)). Particle velocity increased almost linearly with ${ }_{10}$ the average input fluid velocity. Its value was greater than the average fluid velocity (blue dashed line), but less than the maximum velocity of the fluid (red dashed line). This is because the particles were focused at the centre of the channel along the long face where the fluid velocity was maximum, while along the short face, due to strong shear-gradient induced lift force, the particles were actually focused half way between the axis of the channel and the top/bottom surface (two blue dots in Fig. 5b(ii)), which agrees well with our prediction. Although our proposed serpentine channel can only focus ${ }_{15}$ particles in 2-D manner (a single focusing streak from top view), focusing particles in 3-D can easily be implemented after careful adjustment, such as (i) reducing channel height to less than 2 times of particle diameter so that only one particles can occupy short face or (ii) placing several grooves on the bottom of serpentine channel at the outlet to induce rotating flows to focus particles in 3D (Chung et al. 2013).

\section{Conclusions}

${ }_{20}$ The present work presented a new concept of inertial focusing which only takes account of secondary flow drag and particle centrifugal force. The focusing mechanism and design considerations were proposed, which provide useful guidelines for the design of curved channel for particle focusing. Numerical modelling based on the proposed focusing concept was conducted, and the numerical results agreed well with experimental ones. An extremely low aspect ratio (1/5) 
channel was used to suppress the mixing effects of counter-rotating secondary flows, as well as keeping its positive effects for particles focusing in a serpentine channel. Effective particle focusing at the centre of a symmetric serpentine channel was available at a wide range of flow conditions. Compared with an asymmetric serpentine channel, a symmetric serpentine channel has a much simpler structure, and particle focusing at the centre of the channel is convenient for flow ${ }_{5}$ cytometry applications. The effect of particle centrifugal force on the particle focusing was evaluated. While we disagreed with previous reports that focusing in a curving channel was independent of solution density and particle centrifugal force, we found that particle centrifugal force played a significant role on particle focusing, which needs to be taken into account in practical applications. Focusing can be obtained within a state-of-the-art length, with the significant advantage of lower fluidic resistance. In conclusion, our study not only provides a simple focusing unit which can potentially be applied for ${ }_{10}$ particle enrichment, filtration and flow cytometry, it also extends our understanding of the effects of particle centrifugal force on the particle focusing process in a curving channel.

\section{Acknowledgements}

This work is partially supported by the University of Wollongong through a UIC grant. The authors thank Professor Dino Di Carlo, University of California, Los Angeles for the helpful discussions.

\section{References}

ASMOLOV ES (1999) The inertial lift on a spherical particle in a plane Poiseuille flow at large channel Reynolds number. J Fluid Mech 381:63-87. Bhagat AAS, Bow H, Hou HW, Tan SJ, Han J, Lim CT (2010a) Microfluidics for cell separation. Med Biol Eng Comput 48 (10):999-1014.

Bhagat AAS, Kuntaegowdanahalli SS, Kaval N, Seliskar CJ, Papautsky I (2010b) Inertial microfluidics for sheath-less high-throughput flow cytometry. Biomed Microdevices 12 (2):187-195.

20 Bhagat AAS, Kuntaegowdanahalli SS, Papautsky I (2008) Continuous particle separation in spiral microchannels using dean flows and differential migration. Lab Chip 8:1906-1914.

Bhagat AAS, Kuntaegowdanahalli SS, Papautsky I (2009) Inertial microfluidics for continuous particle filtration and extraction. Microfluid Nanofluid 7 (2):217-226.

Çetin B, Li D (2011) Dielectrophoresis in microfluidics technology. Electrophoresis 32 (18):2410-2427.

${ }_{25}$ Chung AJ, Gossett DR, Di Carlo D (2013) Three Dimensional, Sheathless, and High-Throughput Microparticle Inertial Focusing Through Geometry-Induced Secondary Flows. Small 9 (5):685-690.

Cristofanilli M, Budd GT, Ellis MJ, Stopeck A, Matera J, Miller MC, Reuben JM, Doyle GV, Allard WJ, Terstappen LWMM (2004) Circulating tumor cells, disease progression, and survival in metastatic breast cancer. N Engl J Med 351 (8):781-791.

Di Carlo D (2009) Inertial microfluidics. Lab Chip 9:3038-3046.

30 Di Carlo D, Irimia D, Tompkins RG, Toner M (2007) Continuous inertial focusing, ordering, and separation of particles in microchannels. Proc Natl Acad Sci USA 104 (48):18892.

Di Carlo D, Jon F, Irimia D, Tompkins RG, Toner M (2008) Equilibrium separation and filtration of particles using differential inertial focusing. Anal Chem 80 (6):2204-2211.

Duffy DC, McDonald JC, Schueller OJA, Whitesides GM (1998) Rapid prototyping of microfluidic systems in poly (dimethylsiloxane). Anal Chem 70 35 (23):4974-4984.

Eisenstein M (2006) Cell sorting: divide and conquer. Nature 441 (7097):1179-1185.

Forbes TP, Forry SP (2012) Microfluidic magnetophoretic separations of immunomagnetically labeled rare mammalian cells. Lab Chip 12 (8):1471-1479.

Gossett DR, Carlo DD (2009) Particle focusing mechanisms in curving confined flows. Anal Chem 81 (20):8459-8465.

Hansson J, Karlsson JM, Haraldsson T, Brismar H, van der Wijngaart W, Russom A (2012) Inertial microfluidics in parallel channels for high-throughput applications. Lab Chip 12:4644-4650.

Hou HW, Warkiani ME, Khoo BL, Li ZR, Soo RA, Tan DS-W, Lim W-T, Han J, Bhagat AAS, Lim CT (2013) Isolation and retrieval of circulating tumor cells using centrifugal forces. Scientific reports 3:1259.

Huang LR, Cox EC, Austin RH, Sturm JC (2004) Continuous particle separation through deterministic lateral displacement. Science 304 (5673):987-990.

Hur SC, Tse HTK, Di Carlo D (2010) Sheathless inertial cell ordering for extreme throughput flow cytometry. Lab Chip 10:274-280.

45 Ji HM, Samper V, Chen Y, Heng CK, Lim TM, Yobas L (2008) Silicon-based microfilters for whole blood cell separation. Biomed Microdevices 10 (2):251-257.

Kuntaegowdanahalli SS, Bhagat AAS, Kumar G, Papautsky I (2009) Inertial microfluidics for continuous particle separation in spiral microchannels. Lab Chip 9:2973-2980.

Lee MG, Choi S, Kim HJ, Lim HK, Kim JH, Huh N, Park JK (2011a) Inertial blood plasma separation in a contraction-expansion array microchannel.

50 Appl Phys Lett 98 (25):253702-253702-253703.

Lee MG, Choi S, Park JK (2009a) Rapid laminating mixer using a contraction-expansion array microchannel. Appl Phys Lett 95 (5):051902-051902051903.

Lee MG, Choi S, Park JK (2009b) Three-dimensional hydrodynamic focusing with a single sheath flow in a single-layer microfluidic device. Lab Chip 9:3155-3160.

55 Lee WC, Bhagat AAS, Huang S, Van Vliet KJ, Han J, Lim CT (2011b) High-throughput cell cycle synchronization using inertial forces in spiral microchannels. Lab Chip 11:1359-1367. 
Lim DSW, Shelby JP, Kuo JS, Chiu DT (2003) Dynamic formation of ring-shaped patterns of colloidal particles in microfluidic systems. Appl Phys Lett 83 (6):1145-1147.

Mach AJ, Kim JH, Arshi A, Hur SC, Di Carlo D (2011) Automated cellular sample preparation using a Centrifuge-on-a-Chip. Lab Chip 11:2827-2834.

Martel JM, Toner M (2012) Inertial focusing dynamics in spiral microchannels. Phys Fluids 24:032001.

${ }_{5}$ Moon HS, Kwon K, Kim SI, Han H, Sohn J, Lee S, Jung HI (2011) Continuous separation of breast cancer cells from blood samples using multi-orifice flow fractionation (MOFF) and dielectrophoresis (DEP). Lab Chip 11:1118-1125.

Oakey J, Applegate Jr RW, Arellano E, Carlo DD, Graves SW, Toner M (2010) Particle focusing in staged inertial microfluidic devices for flow cytometry. Anal Chem 82 (9):3862-3867.

Oozeki N, Ookawara S, Ogawa K, Löb P, Hessel V (2009) Characterization of microseparator/classifier with a simple arc microchannel. AIChE J 55 $10 \quad$ (1):24-34.

Park JS, Song SH, Jung HI (2009) Continuous focusing of microparticles using inertial lift force and vorticity via multi-orifice microfluidic channels. Lab Chip 9:939-948.

Richardson JF, Coulson JM, Harker J, Backhurst J (2002) Chemical Engineering: Particle technology and separation processes, vol 2. ButterworthHeinemann.

15 Russom A, Gupta AK, Nagrath S, Di Carlo D, Edd JF, Toner M (2009) Differential inertial focusing of particles in curved low-aspect-ratio microchannels. New journal of physics 11 (7):075025.

Segre G (1961) Radial particle displacements in Poiseuille flow of suspensions. Nature 189:209-210.

Segre G, Silberberg A (1962) Behaviour of macroscopic rigid spheres in Poiseuille flow Part 2. Experimental results and interpretation. J Fluid Mech 14 (01):136-157.

20 Seo J, Lean MH, Kole A (2007a) Membrane-free microfiltration by asymmetric inertial migration. Appl Phys Lett 91 (3):033901-033901-033903.

Seo J, Lean MH, Kole A (2007b) Membraneless microseparation by asymmetry in curvilinear laminar flows. J Chromatogr A 1162 (2):126-131.

Stroock AD, Dertinger SKW, Ajdari A, Mezić I, Stone HA, Whitesides GM (2002) Chaotic mixer for microchannels. Science 295 (5555):647.

Sudarsan AP, Ugaz VM (2006) Multivortex micromixing. Proc Natl Acad Sci USA 103 (19):7228-7233.

Vermes I, Weitz DA, van den Berg A (2012) High-yield cell ordering and deterministic cell-in-droplet encapsulation using Dean flow in a curved

25 microchannel. Lab Chip 12:2881-2887.

Wang Z, Zhe J (2011) Recent advances in particle and droplet manipulation for lab-on-a-chip devices based on surface acoustic waves. Lab Chip 11:12801285.

Wu L, Guan G, Hou HW, Bhagat AAS, Han J (2012) Separation of Leukocytes from Blood Using Spiral Channel with Trapezoid Cross-Section. Anal Chem 84 (21):9324-9331.

30 Yamada M, Nakashima M, Seki M (2004) Pinched flow fractionation: continuous size separation of particles utilizing a laminar flow profile in a pinched microchannel. Anal Chem 76 (18):5465-5471.

Yoon DH, Ha JB, Bahk YK, Arakawa T, Shoji S, Go JS (2008) Size-selective separation of micro beads by utilizing secondary flow in a curved rectangular microchannel. Lab Chip 9:87-90.

Zhang J, Li M, Li WH, Alici G (2013) Inertial focusing in a straight channel with asymmetrical expansion-contraction cavity arrays using two secondary flows. J Micromech Microeng 23 (8):085023.

Zheng Z, Fletcher DF, Haynes BS (2013) Laminar heat transfer simulations for periodic zigzag semicircular channels: Chaotic advection and geometric effects. Int J Heat Mass Transfer 62:391-401.

Zhou J, Papautsky I (2013) Fundamentals of inertial focusing in microchannels. Lab Chip 13:1121-1132.

Zhu J, Tzeng T-RJ, Hu G, Xuan X (2009) DC dielectrophoretic focusing of particles in a serpentine microchannel. Microfluid Nanofluid 7 (6):751-756. 